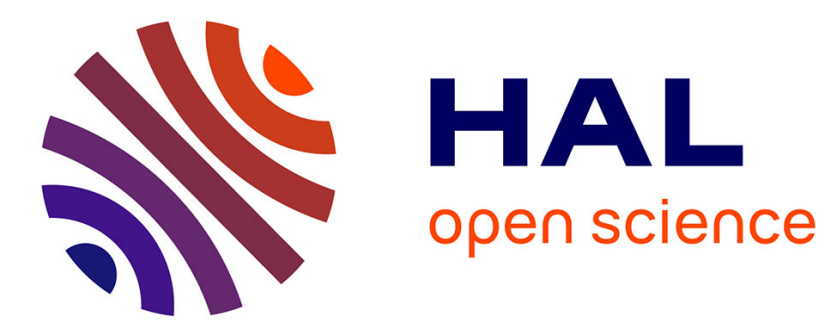

\title{
Étude du spectre solaire ultra-violet
}

A. Cornu

\section{To cite this version:}

A. Cornu. Étude du spectre solaire ultra-violet. J. Phys. Theor. Appl., 1878, 7 (1), pp.285-295. 10.1051/jphystap:018780070028500 . jpa-00237428

\section{HAL Id: jpa-00237428 https://hal.science/jpa-00237428}

Submitted on 1 Jan 1878

HAL is a multi-disciplinary open access archive for the deposit and dissemination of scientific research documents, whether they are published or not. The documents may come from teaching and research institutions in France or abroad, or from public or private research centers.
L'archive ouverte pluridisciplinaire HAL, est destinée au dépôt et à la diffusion de documents scientifiques de niveau recherche, publiés ou non, émanant des établissements d'enseignement et de recherche français ou étrangers, des laboratoires publics ou privés. 


\section{ÉTUDE DU SPEGTRE SOLAIRE OLTRA-VIOLET;}

Par M. A. CORNU.

L'étude des radiations solaires est l'une des branches les plus fécondes de l'Optique et l'une des sources les plus précieuses de nos connaissances sur la constitution du Soleil. Bien que cette étude ait été déjà poussée fort loin par des physiciens éminents, elle offrira encore longtemps un champ fertile d'explorations et conduira à des points de vue nouveaux et à des rapprochements inattendus. C'est ce qui ressortira, je l'espère, du travail que j'ai poursuivi pendant plusieurs années et dont je vais donner un extrait.

Ce travail est la continuation du beau Mémoire d'Angström sur le Spectre normal du Soleil et son extension au delà du spectre visible, auquel le savant suédois s'était arrêté. Il comprend toute la partie ultra-violette de ce spectre; il est résumé par deux planches faisant suite à l'Atlas d'Angström, dans le même format et à la mème échelle.

J'ai divisé ce travail en deux Parlies : la première comprenant la portion du spectre ultra-violet observable avec les spectroscopes ordinaires des laboratoires munis d'objectifs et de prismes en verre; elle s'étend depuis le violet extrême, c'est-à-dire depuis la raie $h^{\prime}(\lambda=410$, ro $)$ jusqu'à la raie $\mathrm{O}(\lambda==343,97)$; le reste des radiations est absorbé par la matière même des objectifs et des prismes. La planche qui résume ce travail a paru dans les Annales de l'École Normale, t. III, 1874.

La deuxième Partie, résumée par une planche actuellement en cours de gravure, s'étend depuis la raie $O$ jusqu'à l'extrémité ultra-violette observable photographiquement à l'aide d'un spectroscope dont les objectifs sont en quartz et le prisme en spath d'Islande : cette limite correspond à la raie $U(\lambda=294,80)$.

Les raies sombres du spectre solaire sont, comme dans l'ensemble du Mémoire, rangées suivant l'échelle normale, c'est-à-dire par ordre de longueur d'onde. J'ai été assez heureux pour étendre d'une manière notable la partie déjà décrite de ce spectre. Les déterminations des longueurs d'onde des raies principales n'ont

J. de Phys., t. VII. (Septembre 1878 .)

2 I 
pas été sans présenter de grandes difficultés, à cause de la faiblesse des radiations de grande réfrangibilité. J'ai toutefois réussi à obtenir celles des raies $\mathrm{O}, \mathrm{P}, \mathrm{Q}, \mathrm{R}, r$ et $\mathrm{S}$ avec la lumière solaire directe, en opérant par réflexion sur un réseau tracé sur verre.

Pour les radiations plus réfrangibles, trop faibles dans la lumière solaire pour être utilisées dans ces déterminations, après m'ètre assuré de la coïncidence des raies sombres de cette région du spectre solaire avec les raies du spectre de l'élincelle du fer (celte cö̈ncidence peut s'établir qualitativement avec une perfection bien supérieure à l'approximation des mesures), j'ai déterminé les longueurs d'onde de ces raies avec la plus grande facilité.

Voici les valeurs qui ont servi de repères pour la construction des planches de la deuxième partie du spectre ultra-violet;

\begin{tabular}{|c|c|c|c|c|c|}
\hline Raic & O. & $\lambda=343,97$ & Raie & $\ldots$ & $\lambda=314,42$ \\
\hline D & P.... & $335,9^{3}$ & $"$ & S. & $309,9^{5}$ \\
\hline " & Q... & 328,49 & ") & $\mathrm{T}$ & 3o, 97 \\
\hline » & R.... & $3 \div 7,9^{\circ}$ & ) & U. & 294,80 \\
\hline
\end{tabular}

Les valeurs relatives aux quatre promières raies s'accordent trèsbien avec celles de M. Mascart, dont j'ai d'ailleurs adopté les notations jusqu'à la raie $\mathrm{S}$; au delà, la faiblesse de l'impression photographique, qui avait empêché M. Mascart de pousser plus loin que $\mathrm{R}$ la détermination des longueurs d'onde, se fait sentir dans le dessin que cet habile physicien a donné comme résumé de son travail, du moins dans la partie voisine de la limite observable.

Ainsi déjà la raie $\mathrm{S}$ donnée comme simple est au moins double; aussi ai-je préféré adopter comme repère la grosse raie immédiatement contiguë; elle est cependant triple, mais elle est très-précieuse, parce que scs trois composantes sont égales et équidistantes; de plus elle correspond exactement à une raie très-brillante du spectre de la vapeur de fer, qui, dans l'arc électrique, se résout en trois raies excessivement nettes. En nommant $\mathrm{S}_{2}$ ce groupe, il n'y aura aucune confusion possible.

Quant à la raie $T$, qui forme la limite de la région observée par M. Mascart, je n'ai pu établir avec certitude la correspondance de son dessin avec mes clichés; j’ai donc donné ce nom à une très- 
grosse raie duuble dont l'importance est plus considérable, et qui a de plus l'avantage, comme la raie $\mathrm{S}_{2}$, de correspondre à une raie double très-brillante du spectre de la vapeur de fer.

Enfin, à la limite de la région que j'ai pu observer, se rencontre une raie sombre correspondant aussi à une raie importante $\dot{\alpha} u$ fer, et pour laquelle je propose provisoirement la dénomination de $\mathbf{U}$.

Pour rendre plus claire cette description de la partie nouvelle du spectre ultra-violet, voici un dessin comprenant les raies simples principales, celles qui donnent à cette partie du spectre sa physionomie propre.

Fig. I.

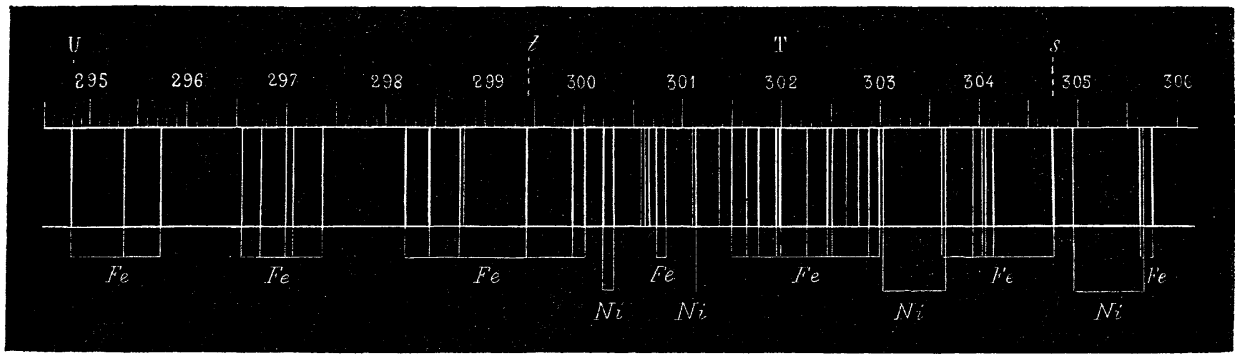

Cette figure est extraite du dessin détaillé dont il a étć question

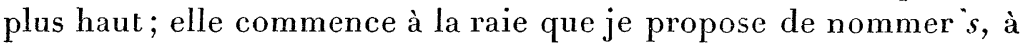
cause de la facilité avec laquelle elle se distingue des autres cntre les raies $\mathrm{S}$ et $\mathrm{T}$, et se termine à la limite de visibilité du spectre solaire.

Cette limite ne dépend plus, comme dans le cas des spectroscopes ordinaires construits en crown-glass ou en flint-glass, du pouvoir absorbant des milieux transparents employés, puisque l'appareil est construit en quartz et en spath d'lslande. On s'en assure aisément par l'observation photographique des radiations brillantes des spectres métalliques dont la réfrangibilité s'étend extrêmement loin. Pour fixer les idécs, on sait qu'avec un prisme de spath d'Islande (rayon ordinaire) d'un angle de $60^{\circ}$, 'on peut observer des radiations déviées de plus de $70^{\circ} ;$ avec les radiations solaires, les impressions photographiques cessent d'êtrc visibles pour une déviation d'environ $60^{\circ}$. 
D'où vient cette limitation du spectre solaire dans la région ultraviolette? Une série d'études préliminairesm'a permis de reconnaître la cause de cette limitation spontanée du spectre solaire et de déterminer les conditions les plus favorables à l'extension de cette limite. Cette cause n'est autre que l'absorption atmosphérique; il suffit, en effet, d'observer méthodiquement l'impression photographique du spectre solaire ultra-violet pendant toute une journée pour reconnaître que l'étendue du spectre varie dans le même sens que la hauteur du Soleil au-dessus de l'horizon. L'expérience montre que le maximum d'étendue se présente entre $\mathrm{I}^{\mathrm{b}}$ du matin et $\mathrm{I}^{\mathrm{b}} 3 \mathrm{o}^{\mathrm{m}}$ de l'après-midi.

Comme on devait s'y attendre, c'est à l'époque du solstice d'été qu'on obtient le maximum maximorum d'étendue du spectre : c'est par des observations effectuées à cette époque de l'année que j'ai pu étendre jusqu'à la raie U la description du spectre représenté ci-dessus; mais ce qu'on ne pouvait guère prévoir, c'est l'étendue du spectre qu'on obtient encore en hiver, mème dans l'atmosphère embrumée de Paris, à l'époque du solstice d'hiver; des clichés obtenus récemment ont donné, un peu après-midi, des impressions photographiques qui atteignent presque la raie $T$.

Il résulte de ces faits la conséquence très-curieuse, qu'à égalité de hauteur du Soleil le spectre solaire observé est notablement plus étendu en hiver qu'en été.

Ce résultat s'explique d'une manière très-simple, si l'on attribue à la vapeur d'eau contenue dans l'atmosphère le pouvoir absorbant qui limite le spectre solaire ultra-violet. En effet, la quantité de vapeur d'eau contenue par mètre cube dans l'atmosphère est beaucoup plus grande en été qu'en hiver. Si l'on adopte l'état hygrométrique moyen 0,75 , la pression moyenne $760^{\mathrm{mm}}$, la température zéro pour le solstice d'hiver et $30^{\circ}$ pour la température moyenne à midi au solstice d'été, on trouve respectivement $3^{\mathrm{gr}}, 6$ et $25^{\mathrm{gr}}$, o, c'est-à-dire qu'il existe près de sept fois plus de vapeur d'eau dans les basses régions de l'atmosphère terrestre en été qu'en hiver.

Cette action de la vapeur d'eau sur les radiations solaires a d'ailleurs été invoquée par plusieurs physiciens (Angström, Janssen) dans l'étude du spectre visible, pour l'explication des raies et bandes désignées quelquefois sous le nom de raies atmo- 
sphériques. Dans le spectre ultra-violet, cette absorption ne parait pas localisée sous forme de raies ou de bandes; l'action est continue sans maxima appréciables.

Il reste à dire quelques mots des autres perfectionnements expérimentaux qui m'ont permis d'aller plus loin que mes devanciers, comme étendue du spectre et comme finesse de détails; en voici l'énumération :

$1^{\circ}$ La substitution d'un prisme à réflexion totale en quartz au miroir métallique de l'héliostat;

$2^{\circ}$ Le calcul rationnel des courbures des lentilles de quartz du spectroscope, par la condition de donner le minimum d'aberration pour une grande ouverture angulaire $\left(\frac{1}{10}\right)$;

$3^{\circ}$ L'égalité d'épaisseur et le signe contraire du pouvoir rotatoire des deux lentilles de quartz : on compense ainsi certaines aberrations;

$4^{\circ}$ La perfection de taille des faces du prisme de spath d'Islande;

$5^{\circ}$ L'emploi d'une lentille de quartz concentrant les rayons solaires sur la fente du spectroscope : cette addition, loin de troubler les images, comme cela a lieu lorsque l'aplanétisme des diverses parties du spectroscope, ou leur réglage optique, n'est pas trèscomplet, apporte un accroissement considérable dans l'intensité des impressions photographiques et une grande perfection de détails, parce qu'on peut resserrer la fente sans cesser d'avoir l'éclat nécessaire à la formation des clichés.

Cette description des raies sombres du spectre solaire a été complétée par l'étude comparative des raies brillantes des vapeurs métalliques : les résultats obtenus me paraissent d'un grand intérêt au point de vue de l'Astronomie physique.

On sait que dans le spectre visible du Soleil la presque totalité des raies sombres correspond exactement à des raies brillantes des spectres des vapeurs métalliques; ce renversement dans l'apparence des raies n'est qu'un effet de contraste et s'explique par l'existence sur le Soleil d'une couche de vapeurs à une température relativement basse, absorbant partiellement les radiations du spectre continu d'un fond plus brillant. L'étude comparative de ces spectres a constitué une vraie méthode d'analyse qualitative et a conduit à mettre hors de doute l'existence sur le Soleil d'un certain nombre d'éléments chimiques terrestres. 
L'extension de cette étude aux raies sombres du spectre ultraviolet, en agrandissant le champ de comparaison, m'a permis d'aller plus loin dans cette voie et d'aborder, jusqu'à un certain point, l'analyse quantitative des éléments de cette couche absorbante, à l'action de laquelle les raies sombres du spectre solaire sont attribuées. Voici le résumé de cette étude :

On remarque tout d'abord que les groupes de raies intenses sont inégalement répartis sur toute l'étendue du spectre solaire: la partie la moins réfrangible n'en contient à peu près aucun (en mettant à part les bandes el raies atmosphériques); ce n'est qu'à partir du bleu indigo que commencent les groupes sombres dont le groupe G est un type; on rencontre ensuite les deux raies $\mathrm{H}, \mathrm{K}$, larges et estompées, qui se détachent sur un fond relativement clair, puis les groupes très-sombres $\mathrm{L}, \mathrm{M}, \mathrm{N}, \mathrm{O}, \mathrm{P}$; au delà vient un espace plus clair encore, sur lequel se détache la raie $Q$, puis les groupes voisins de R et de $r$; un nouvel espace assez brillant conduit aux groupes sombres $\mathrm{S}, \mathrm{T}, \mathrm{U}$.

L'examen comparatif des spectres des vapeurs métalliques montre de prime-abord que ces masses de raies sombres correspondent en général aux raies brillantes du spectre de la vapeur de fer, qui comprend à lui seul la presque totalité des groupes $\mathrm{G}, \mathrm{L}, \mathrm{M}, \mathrm{N}, \mathrm{O}$, $\mathrm{Q}, \mathrm{S}, \mathrm{T}, \mathrm{U}$ et plusieurs des groupes voisins de R.

Les deux grosses raics $\mathrm{H}$ et $\mathrm{K}$ correspondent au calcium, ainsi que deux raies analogues constituant $\mathrm{R}$ et le groupe compris entre $\mathrm{R}$ et $r$.

C'est au nickel que se rapportent la plupart des raies importantes, comprises entre $\mathrm{O}$ et $\mathrm{P}$, ainsi qu'un nombre très-notable de raies dans la région STU (voir la figure précédente).

Les autres métaux magnétiques, cobalt, manganèse, chrome, fournissent des raies de moindre importance; le titane présente un très-grand nombre de coïncidences, mais avec des raies en général très-faibles, excepté entre $Q$ et $R$, où leur importance est plus grande; l'étain offre des coïncidences qui, malgré leur petil nombre, paraissent non équivoques.

Le magnésium fournit quatre raies triples d'apparence identique, que je nommerai $b$ (Fraunhofer) dans le vert, $b^{\prime}$ entre K et L, $b^{\prime \prime}$ entre $\mathrm{P}$ et $\mathrm{Q}$, et $b^{\prime \prime \prime}$ entre $\mathrm{S}$ et $\mathrm{T}$; elles sont, à l'exception de $b^{\prime \prime}$, qui est relativement faible dans le spectre du magnésium, trèsintenses dans le spectre solaire. 
L'aluminium donne deux raies fort nettes entre $\mathrm{H}$ et $\mathrm{K}$, et deux autres analogues entre $\mathrm{S}$ et $\mathrm{T}$. Le sodium qui produit la raie $\mathrm{D}$ du spectre visible ne donne, dans l'ultra-violet, qu'une raie double assez pâle entre $\mathrm{P}$ et $\mathrm{Q}$. Enfin le glucinium paraît être représenté par quelques raies faibles.

Tels sont, en y joignant l'hydrogène qui donne quatre raies sombres $\mathrm{C}, \mathrm{F}, \mathrm{G}^{\prime}$ (près de $\mathrm{G}$ ) et $h$, les éléments chimiques fournissant les coüncidences les plus remarquables avec les raies du spectre solaire.

Le caractère général des groupes de raies sombres du spectre solaire correspondant à un même métal est de présenter une intensité relative tout à fait en rapport avec l'éclat des raies brillantes correspondantes du spectre métallique : il y a donc une véritable proportionnalité entre le pouvoir émissif des vapeurs métalliques incandescentes et leur pouvoir absorbant, ce qui est d'ailleurs la base de l'explication du renversement des raies solaires (Kirchhoff, Foucault, Angström, Stockes). Si l'on joint à cette remarque la considération de l'éclat intrinsèque moyen du spectre de chaque élément chimique dans les régions à comparer, on arrive à conclure que l'intensité des raies sombres du spectre solaire est caractéristique de la quantité relative des différentes vapeurs métalliques qui, à la surface du Soleil, sont la cause de ces raies sombres. L'établissement d'une méthode d'analyse quantitative fondée sur ces considérations exigerait encore bien des efforts; mais, si l'on cherche seulement à se rendre un compte approché de la composition de cette couche absorbante qui forme l'enveloppe extérieure du Soleil, les observations présentes suffisent pour une première approximation.

Dans cette manière de voir, la vapeur de fer serait de beaucoup plus abondante, à cause du nombre et surtout de l'intensité des raies sombres qui lui correspondent dans le spectre solaire.

Le nickel et le magnésium viendraient en second lieu; le calcium, dont le spectre possède un éclat intrinsèque si grand pour les deux raies $\mathrm{H}$ et $\mathrm{K}$ qui le caractérisent, doit entrer dans une proportion moindre que J'intensité de ces raies ne pourrait le faire supposer; viennent ensuite l'aluminium, le sodium et l'hydrogène, enfin le manganèse, le cobalt, le titane, le chrome et l'étain.

Telle serait approximativement la liste, par ordre de quantité, 
des éléments volatilisés à la surface du Soleil. En examinant cette liste, où le fer, le nickel et le magnésium jouent un si grand rôle, on est immédiatement frappé de l'analogie de cette composition avec celle des aérolithes, dont la majeure partie est formće de fer allié à $\frac{1}{10}$ de nickel : dans les fers météoriques, cet alliage est presque pur; dans les météorites pierreuses, le fer nickelé est mêlé à des silicates magnésiens de compositions diverses.

Cette étude du spectre conduit donc à la conclusion suivante :

La position et l'éclat relatif des raies sombres du spectre solaire s'expliquent par l'action d'une couche absorbante existant sur le Soleil, couche dont la composition serait analogue à celle d'aérolithes volatilisés.

Les conséquences de ce fait, révélé par l'analyse spectrale des radiations solaires, touche d'une manière si directe aux grands problèmes de la Physique cosmique et de l'Astronomie, qu'il me paraît utile de les indiquer, avec toute la réserve que comporte d'ailleurs un sujet aussi délicat.

I Probabilité d'une action magnétique directe sur le Soleil. - Si la couche extérieure du Soleil contient, comme les aérolithes, une proportion considérable de vapeurs de fer, la masse absolue de ce métal répartie sur la surface du Soleil doit être trèsgrande, vu l'énorme diamètre de cet astre, et doit exercer une action appréciable sur les phénomènes magnétiques terrestres.

On pourrail objecter que le fer, porté à l'incandescence, perd son action attractive sur l'aiguille aimantée; cette diminution rapide avec la température a, en effet, été constatée, mais il n'est aucunement prouvé que ce qui reste de pouvoir magnétique soit rigoureusement nul; il suffirait que le magnétisme spécifique de la vapeur de fer fût de l'ordre de l'attraction newtonienne, pour que l'action magnétique de la masse ferrugineuse du Soleil fût encore très-appréciable sur la Terre.

Dans cette manière de voir, les variations diurnes de l'aiguille aimantée seraient dues à l'action magnétique directe du Soleil. Cette opinion, d'ailleurs, n'est pas nouvelle; elle a été soutenue par des physiciens éminents, en particulier par le général Sabine, dans sa belle publication des observations magnétiques organisées par lui à la surface du globe. La relation des variations moyennes 
avec la position du Soleil aux diverses heures du jour et aux différentes latitudes ressort, avec une évidence manifeste, de la discussion des observations. Par des considérations d'un tout autre ordre, la spectroscopie apporte une confirmation de cette opinion.

$2^{\circ}$ Probabilité en faveur de l'hypothèse de l'aimant terrestre. - La présence d'une quantité considérable de fer dans la composition du Soleil conduit à se demander si cette particularité est purement accidentelle ou si tous les corps du système solaire (et peut-être tous les corps sidéraux ) n'auraient pas une origine commune qui se révélerait par la présence, dans une proportion notable, de fer que le spectroscope a décelé dans l'enveloppe extérieure du Soleil.

Le globe terrestre présente en faveur de cette idée un argument bien sérieux : en effet, la densité moyenne du globe, égale à 5,5, est le double à peu près de la densité moyenne des éléments qui en forment la croûte superficielle : on est donc forcé d'admettre, vu la haute température probable des couches intérieures, que la partie centrale de la Terre est constituée par des matières beaucoup plus denses que les matériaux pierreux, conséquemment par des masses métalliques. Si, d'autre part, on considère la force directrice de l'aiguille aimantée sur les différents points du globe et la symétrie approchée de l'ensemble de ses positions avec certains grands cercles de la sphère terrestre, on est amené à conclure avec une grande probabilité que les masses métalliques du centre de la Terre sont constituées, en proportion notable, par du fer métallique.

Notre satellite exerce aussi sur l'aiguille aimantée une action assez faible, il est vrai, mais qui paraît non équivoque : on pourrait donc voir dans cette action une preuve de l'existence du fer dans la composition moyenne de cet astre.

Enfin la profusion des aérolithes dans notre système planétaire tendrait à confirmer l'idée d'une commune origine de tous les corps célestes (hypothèse cosmogonique de Laplace) et à faire voir dans ces aérolithes le type de la matière cosmique élémentaire.

$3^{\circ}$ Probabilité en faveur de l'origine électrique de la lumière émise par les protubérances solaires. - Les conséquences précédentes se déduisent de la présence pour ainsi dire statique de masses magnétiques à la surface du Soleil. Examinons ce qui peut 
arriver si ces masses sont à l'état de mouvement. Cet état de mouvement existe, ainsi que le prouvent les observations directes de cet astre, et la cause de cet état dynamique réside évidemment dans la chaleur des couches inférieures et dans le refroidissement des couches externes; car, quel que soit le mécanisme de ces mouvements, les conditions thermodynamiques nécessaires à la transformation de la chaleur en force vive sont remplies. Si l'on fait intervenir la condition que certaines parties de ces masses de vapeurs en mouvement sont magnétiques, on voit apparaître la nécessité d'un phénomène secondaire qui semble devoir jouer un rôle important, à savoir la production de courants d'induction dans les masses conductrices avoisinantes, soit en repos, soit animées d'autres mouvements que celles-ci. Il en serait absolument de même si les masses en mouvement relatif, au lieu d'être magnétiques, étaient électrisées par une cause quelconque, en parliculier par quelque action chimique.

Quoiqu'il soit actuellement presque impossible de préciser le mécanisme de ces transformations el d'assigner a priori l'extension et l'énergie de ces phénomènes secondaires, les conditions thermodynamiques sont si farorables, qu'on doit leur supposer une très-grande intensité, et prévoir que certains points au moins de la surface solaire sont le siége de mouvements rapides, et que les masses gazeuses voisines reçoivent, par la propagation des courants induits, un accroissement notable de leur degré d'incandescence.

L'observation des protubérances, tangentiellement au disque solaire, nous révèle précisément l'existence de couches gazeuses dont l'éclat décèle une incandescence plus énergique que celle qui correspondrait à leur position : l'analyse de leur lumière montre que leur spectre est identique à celui de l'hydrogène très-raréfié rendu incandescent par une décharge électrique. La similitude spectrale est complète, tant pour l'éclat relatif des raies brillantes que pour leur netteté décroissant avec la réfrangibilité. Ces protubérances, qui apparaissent dans le voisinage des facules et des taches, ne représenteraient-elles pas ces masses gazeuses traversées par les courants d’induction dans le voisinage des régions magnétiques ou électriques en mouvement rapide?

Cette assimilation aurait l'avantage de faire rentrer dans les conditions thermodynamiques ordinaires l'explication des protubé- 


$$
\text { D. GERNEZ. - ÉBULLition. }
$$

rances, en les présentant comme l'équivalent de l'illumination par induction des masses gazeuses raréfiées, illumination qui se reproduit si aisément dans nos laboratoires avec les plus faibles actions mécaniques. On comprendrait alors la rapidité de l'extension de ces protubérances, leur disparition subite aussi bien que leur permanence accidentelle, sans avoir recours à l'hypothèse de ces jets gazeux doués de vitesses invraisemblables, atteignant plusieurs centaines de kilomètres par seconde. Je dois ajouter que l'hypothèse de vitesses si extraordinaires a déjà été critiquée par M. Fizeau, qui a signalé l'analogie probable du phénomène lumineux des protubérances avec celui que présentent sur notre globe les aurores boréales.

Quelques-unes des conclusions précédentes sembleront peutêtre prématurées; je les soumets néanmoins avec confiance à l'examen des physiciens et des astronomes, comme.le résumé d'un ensemble de faits très-étendus que l'exiguïté de la présente Note ne m'a pas permis de développer ( $\left.{ }^{1}\right)$. 